Am. J. Trop. Med. Hyg., 44(3), 1991, pp. 299-305 (90-203)

Copyright $\odot 1991$ by The American Society of Tropical Medicine and Hyziene

\title{
COEXISTENCE OF GP195 ALLELES OF PLASMODIUM FALCIPARUM IN A SMALL ENDEMIC AREA
}

\author{
SOMCHAI JONGWUTIWES, KAZUYUKI TANABE, SHUSUKE NAKAZAWA, \\ HARUKI UEMURA AND HIROJI KANBARA \\ Institute of Tropical Medicine, Nagasaki University; Osaka Institute of Technology, Japan.
}

\begin{abstract}
Dimorphic variations in the genotype of the precursor to Plasmodium falciparum major merozoite surface antigens or $\mathrm{gp} 195$, among wild isolates in a small malaria parasite population were examined using Southern blot hybridization techniques. Hybridization, with DNA fragment probes and oligonucleotide probes derived from variable blocks of known gp195 alleles against 18 wild isolates from Mae Sod district in Thailand, revealed the existence of seven gp195 alleles, two of which were newly identified in this study. In four out of 17 patients, two different alleles coexisted in the circulation. It was furthermore noted that the seven alleles did not occur at the same frequency, but rather several alleles predominated in the population of $\boldsymbol{P}$. falciparum in this small malaria field.
\end{abstract}

The precursor of Plasmodium falciparum major merozoite surface antigens (MSA-1), or gp195, is one of the potential candidates for asexual blood-stage malaria vaccine. ${ }^{\prime}$ It is synthesized during schizogony and is proteolytically processed into several products of a lower molecular weight. Monkeys vaccinated with gp195-derived immunogens showed partial to complete protection against challenge by the parasite. ${ }^{2--5}$ However, gp 195 shows extensive antigenic diversity, ${ }^{6}$ making it difficult for it to be the base of an effective malarial vaccine. Recent sequence studies on the gp195 gene have revealed that it is composed of seven variable blocks separated by either conserved or semi-conserved blocks. ${ }^{7}$ Variations in the gp195 gene among different isolates are, however, not widely polymorphic but appear to be dimorphic. The dimorphism of the gp195 gene has been widely confirmed ${ }^{8} .9$ with the exception of one variant which lacks apparent repeats near the 5' end of the gene. ${ }^{10,} "$ Furthermore, it is suggested that dimorphic alleles are capable of limited genetic exchange at the sexual stage in a mosquito vector, thus creating different genotypes of the gp195 gene (alleles) in the progeny. ${ }^{7}$ So far, several gp195 alleles have been recognized in culture-adapted parasites originating from various continents. ${ }^{7,11}$

Although it is known that gp 195 alleles are not geographically confined, the following questions remain: (1) how many alleles coexist in a small malaria parasite population? (2) can several alleles coexist in a single patient? and (3) do some alleles predominate in the field? Since $8 p 195$ is a strong candidate for a malaria vaccine, we con- sider it necessary to obtain epidemiological data on such questions at the genetic level. Thus we have carried out hybridization studies against 18 wild isolates obtained from a small malaria field in Thailand.

MATERIALS AND METHODS

\section{Source of parasite DNA}

All of the 18 isolates of $P$. falciparum were collected from 17 patients during November 1988 and January 1989 at Mae Sod, a district in Tak Province in northern Thailand. They were cryopreserved as described elsewhere ${ }^{12}$ and transported to Nagasaki where DNA from each isolate was obtained from in vitro culture according to the method of Trager and Jensen. ${ }^{13}$ The DNA was then purified as described previously. ${ }^{14}$ The isolate $\mathrm{Kl}$ from Thailand was used as a reference control.

\section{Hybridization probes and labeling}

Previous studies have shown that the $\mathrm{K} 1$ and MAD20 isolates can be designated as representatives of dimorphic alleles, ${ }^{7}$ (i.e. the gp195 alleles being different at every variable block). Therefore, both K1 - and MAD20-specific DNA fragments were excised from respective plasmid clones as reported elsewhere. ${ }^{14}$ The probes used here were $\mathrm{K} 216$ and M534 fragments from block 6 and K153 and M180 from block 8 (Fig. 1). A 543 base pair fragment, C543, was also derived from conserved block 3 . These fragments were labeled with digoxigenin-dUTP using a nonra- 
(a)

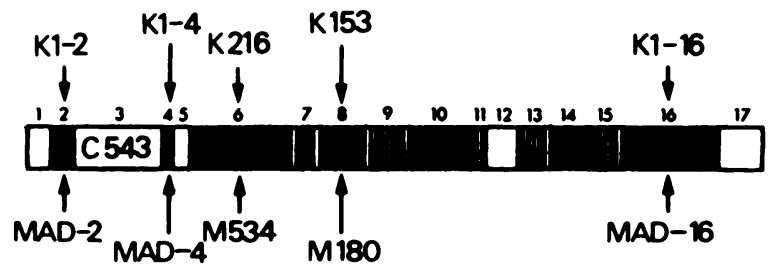

(b)

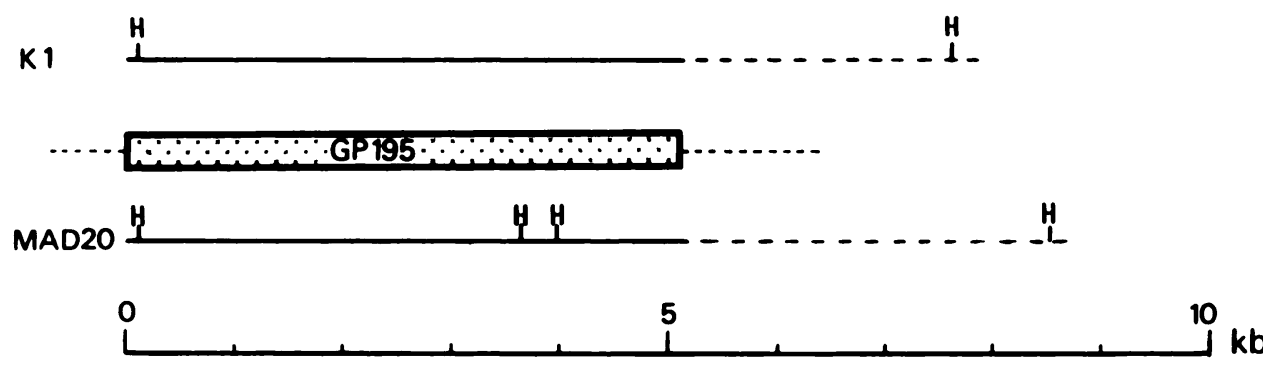

FIOURE 1. (a) Structure of the gp195 gene, ' showing the location of probes used in this study. Sequences are divided into conserved blocks (open boxes), semi-conserved blocks (hatched boxes), and variable blocks (filled boxes). (b) HindIII-restriction sites (H) in and around the gene of the K1 and MAD20 isolates.

dioactive random primed labeling kit according to protocols of the manufacturer (Boehringer Mannheim, FRG).

Five 21-base oligonucleotides and one 30-base oligonucleotide were custom synthesized by Takara Shuzo (Japan): K1-2 (nucleotide residues 217-237), 5'-AGTGGTACAAGTGGTCC AAGT-3'; K1-4 (952-972), 5'-AATCCCCCAC CGGCCAATTCT-3'; K1-16 (4414-4434), 5'-G GAATTGCTGATTTATCAACA-3'; MAD-2 (385-405), 5'-TCAGTTGCTTCAGGTGGTTC A-3'; MAD-4 (1100-1120), 5'-ATGCCGAAAA CCCCACTACTG-3'; MAD-16 (4494-4523), 5'GTTCCCATCATCACCACCAACAACACCTC C-3'. These oligonucleotides were radiolabeled with ${ }^{32} \mathrm{P}$ by phosphorylation with bacteriophage T4 polynucleotide kinase. ${ }^{15}$

\section{Blotting and hybridizations}

Approximately one microgram of parasite DNA was digested completely with $H i n d I I I$ and electrophoresed on $0.8 \%(w / v)$ agarose gels. The DNA was transferred onto a nylon membrane (Hybond-N, Amersham, UK) as described elsewhere. ${ }^{14}$ Procedures for prehybridization, hybridization, washing and immunological detection of the fragment probes were performed following the manufacturer's instructions (Boehringer Mannheim). For the oligonucleotide probes, prehybridization and hybridization were performed at 50; $\mathrm{dgC}$, and membranes were washed four times for $30 \mathrm{~min}$ at 50 ; dgC in 6; ts SSC ( 1 ; ts SSC; eq $0.15 \mathrm{M} \mathrm{NaCl}, 0.015 \mathrm{M}$ trisodium-citrate). The membranes were exposed to Konica X-ray film with intensifying screen for 10-72 hr.

\section{RESULTS}

Southern blots of HindIII-digested DNA of 18 isolates were hybridized with either DNA fragment probes (blocks 3, 6 and 8) or oligonucleotide probes (blocks 2, 4 and 16). These probes gave specific hybridization signals, as shown in eight isolates of $P$. falciparum (Fig. 2). The K1 isolate reacted with $K 1$ probes but not with MAD20 probes, as expected. In the seven wild isolates, all but one (isolate 822 ) were hybridized with either K1 or MAD20 probes, giving signals at the 7.6 or $3.5 \mathrm{~kb}$ region, respectively (see Fig. 1). Both $K 1$ and MAD20 probes from block 6 reacted to 822 and MAD20 probe from block 2 gave two distinct signals. A probe from conserved block 3 also gave two signals at the $\mathbf{7 . 6}$ and $3.5 \mathrm{~kb}$ regions. Results from all hybridization experiments with different probes are summarized in Table 1. Hybridization patterns were identical in some isolates e.g. isolates 807,836 , 842 and 843 . Of 18 isolates, 14 isolates other 

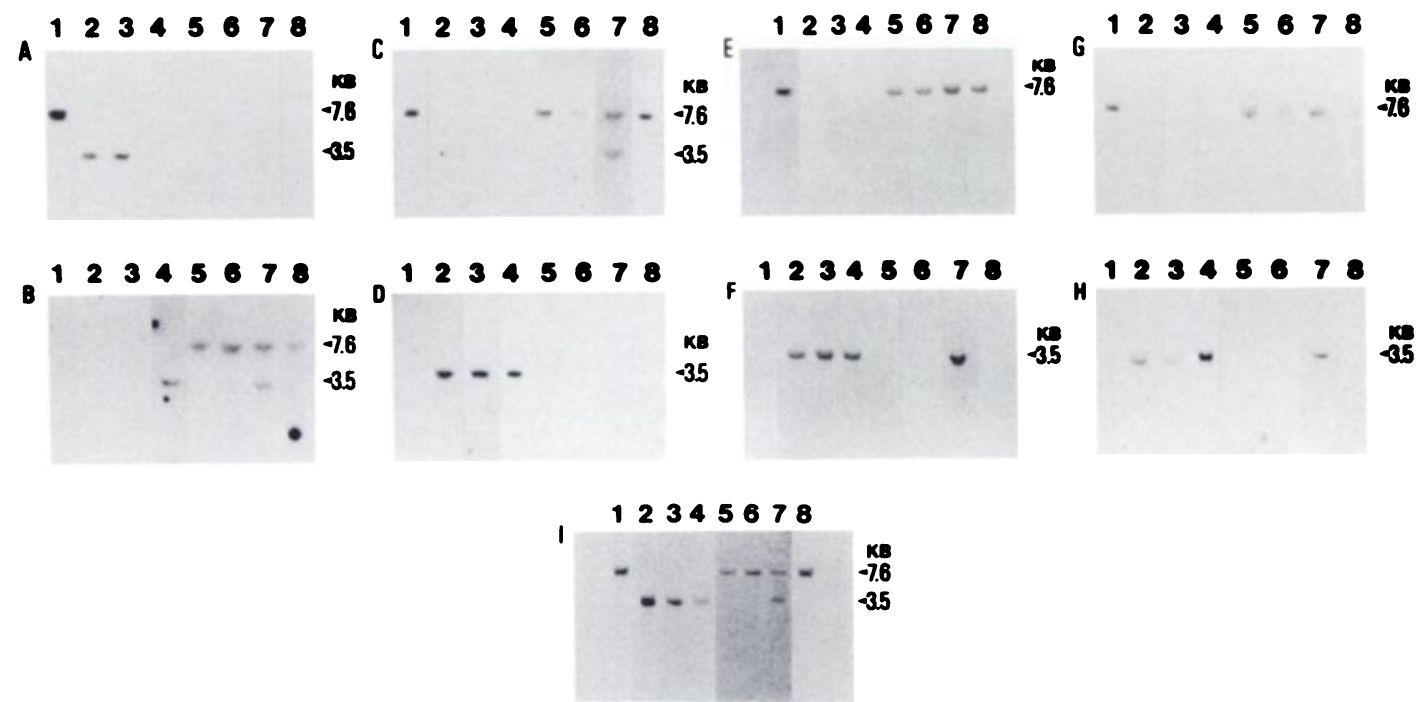

FIGURE 2. Southern blot hybridization of HindIII digested DNA of wild isolates with gp 195-derived probes: A, K1-2 (block 2); B, MAD-2 (block 2); C, K1-4 (block 4); D, MAD-4 (block 4); E, K216 (block 6); F, M534 (block 6); G, K153 (block 8); H, M180 (block 8); I, C543 (block 3). Lanes are: 1, K1; 2, 946; 3, 814; 4, 836; 5, $828 ; 6,827 ; 7,822 ; 8,806$.

than $822,834,835$ and 841 reacted only with either $\mathrm{K} 1$ or MAD20 probe at any given block. The four isolates reacted with both $\mathrm{K} 1$ and MAD20 probes (e.g. blocks 6,8 and 16 in isolates 822) but with only one probe at other blocks (e.g. blocks 2 and 4 in 822). In the latter case, however, each probe gave two distinct signals. Considering the fact that gp195 gene is a single copy gene and the genome of $P$. falciparum is haploid at blood stages, the above results mean that the four isolates $(822,834,835$ and 841$)$ contain two gp195 alleles. Probes from block 2 did not react with 838 . This is probably because 838 lacks an apparent repeat sequence in block 2 and consequently does not hybridize with either oligonucleotide probes. The existence of such variants has been reported in two isolates from Thailand and Ghana. ${ }^{10,11}$

Thus, our hybridization studies identified seven gp195 alleles among 18 wild isolates tested. They are illustrated in Fig. 3 in which isolates are grouped into different alleles. Allele 2 (MAD20 type) shows the genotype which does not share any variable block with allele 1 (K1 type). Alleles 3 to 6 contain both K1 and MAD20 type of variable blocks. It is apparent from the present genotype grouping that recombination occurs between blocks 2,4 and 6 but not between 6,8 and 16 . This confirms previous studies showing limited crossover events at the 5' end portion in the gp195 gene. ${ }^{7.11}$ Furthermore, it is of interest to note that alleles 2 and 3 occur more frequently than other alleles. Figure 3 also lists possible alleles 7 and 8 that were not identified in this study though allele 7 has been reported in isolates from different geographic areas. However, we have identified two new alleles (alleles 5 and 6 ) here. Allele 8 has not yet been found to occur.

\section{DISCUSSION}

Among many malarial antigen genes, the gp 195 gene of $P$. falciparum is unique in the sense that it contains several variable blocks which are principally dimorphic at the genetic level. ${ }^{7,11}$ Previous sequence studies have suggested that recombination of dimorphic gp 195 alleles can create new alleles different from the parental alleles. ${ }^{7,8}$ The recombination, however, seems to be limited, occurring at the 5' end portion of the gene, namely between blocks 2 and 6.', 8.11 The present study confirms this restriction of recombination (see fig. 3). Other workers, using the dot blot hybridization technique against 20 isolates or strains from different parts of the world, have recognized five gp 195 alleles, apart from one allele which apparently lacks repeat region in block 2. They are included in Figure 3. Our hybridization analyses against 18 isolates from a small 


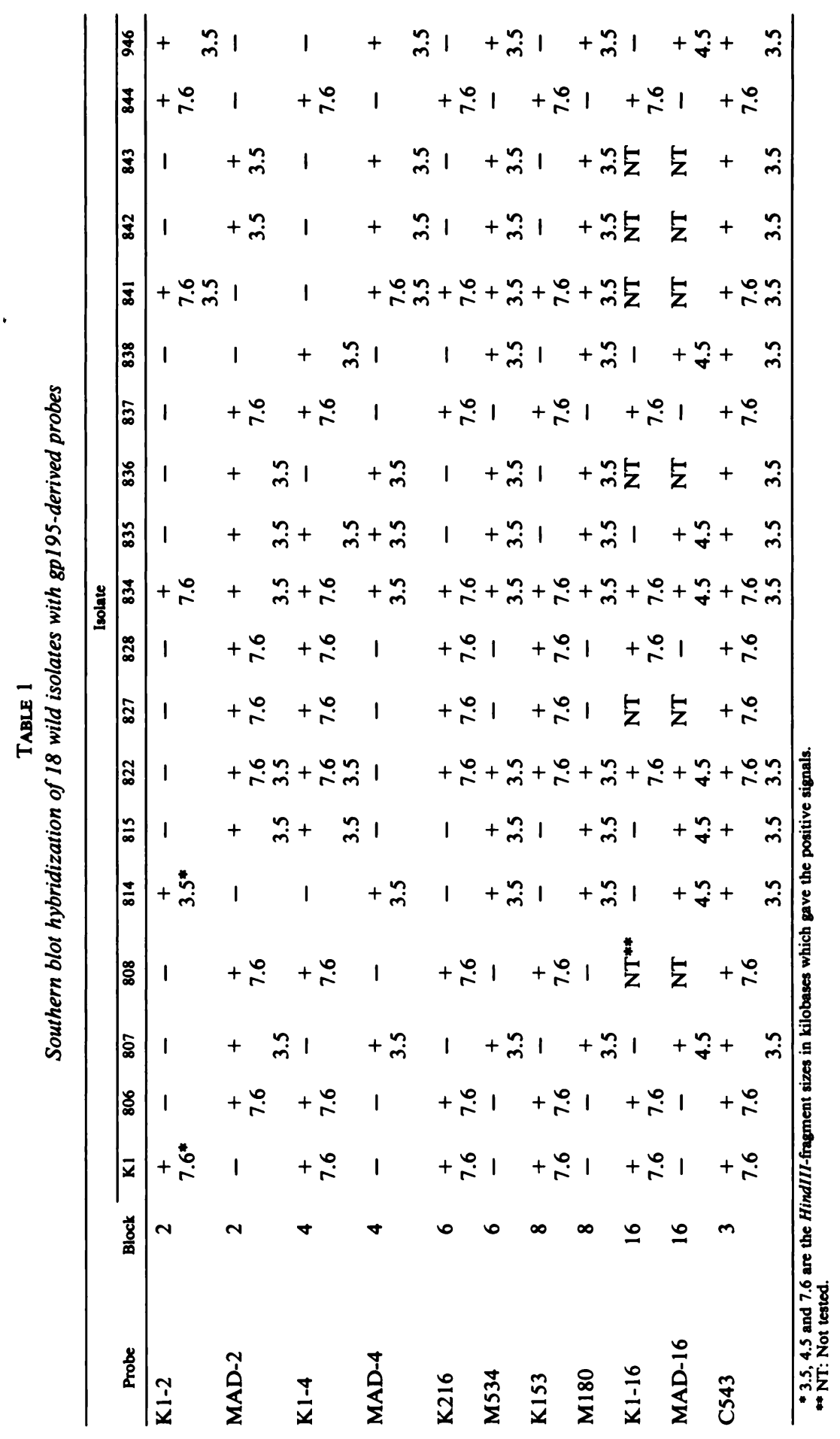




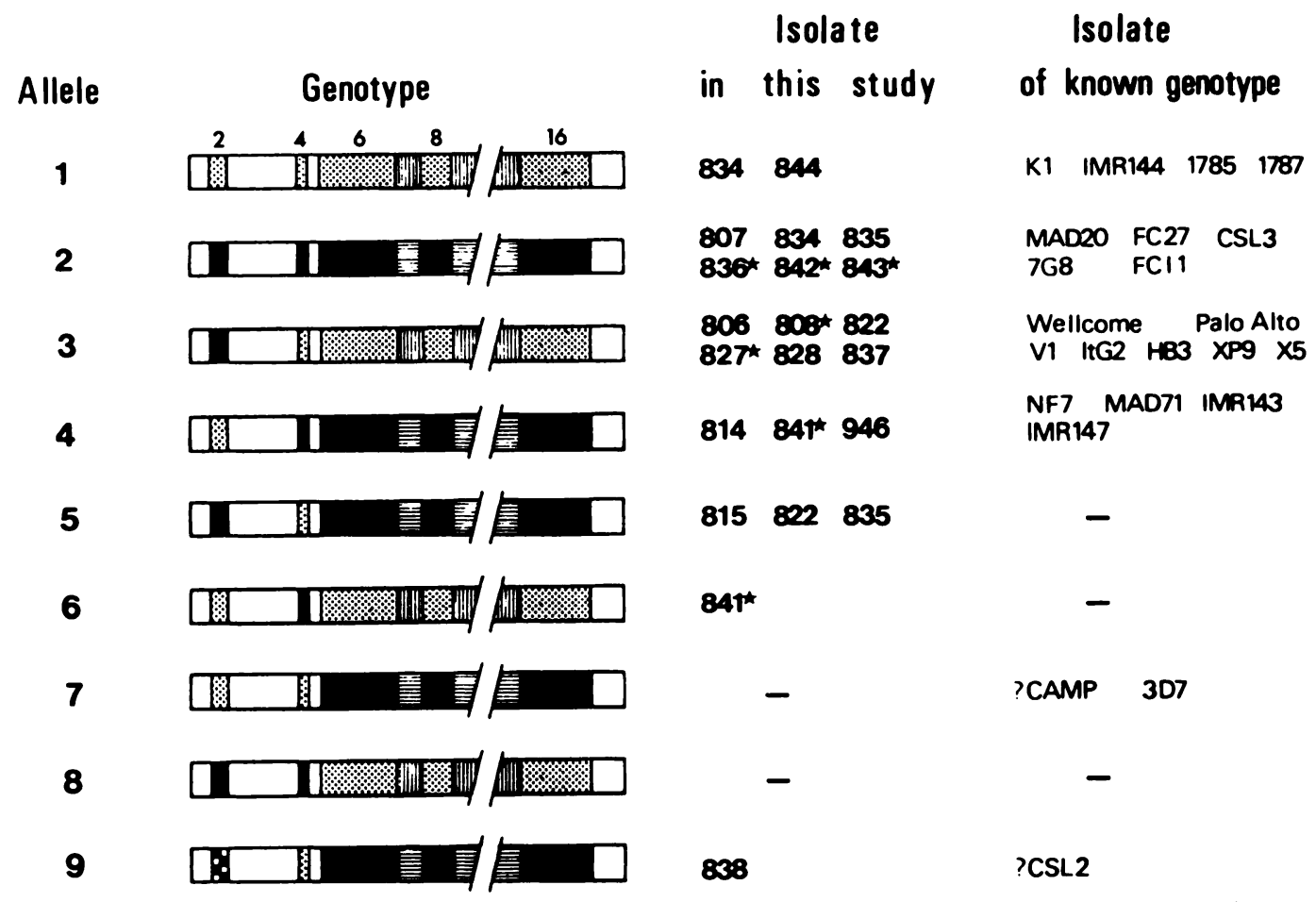

FIGURE 3. Scheme for genotypes of gp195 alleles found in 18 wild isolates studied here and other isolates reported previously." Blocks are: conserved (open boxes); semi-conserved (hatched boxes) and variable (filled or stippled boxes). Isolates marked with an asterisk were not tested for probes of block 16.

malaria field in Thailand have identified seven alleles with two new alleles (alleles 5 and 6).

The coexistence of different gp195 alleles in a localized geographic area has recently been reported by Peterson and others. " They noted three different alleles in eight isolates from the northern coast of Papua New Guinea; alleles 1, 2 and 4 in Figure 3. In the current study, we identified seven alleles. However, the frequency of the occurrence of alleles among the isolates are uneven; alleles 2 and 3 are high in number (6 isolates) while allele 6 is seen as only one, suggesting predominance of several alleles in the Mae Sod district of Thailand. Although the number of isolates examined so far may be insufficient to draw a firm conclusion, this finding may support the notion that genetic diversity of $P$. falciparum shows geographical variation. ${ }^{16}$

Previous serological studies using monoclonal antibodies against gp195 have indicated the coexistence of different serotypes of gp195 in a single malaria patient. ${ }^{17}$ The present study clearly substantiates such coexistence of gp195 at the genetic level. Although we have used uncloned wild isolates, it is widely recognized that in cloned isolates gp195 gene exists in a single copy in the genome of $P$. falciparum. 711,14 Thus, we consider that four out of 18 isolates harbour two genetically distinct clones, and it is unlikely that a single parasite contains two gp195 alleles.

In the present study, parasites were propagated in culture for less than one month. However, in vitro culture of parasites often results in selection of lines that have proliferative advantage. Therefore, if target gene fragments were directly amplified from infected patient material using polymerase chain reaction, the frequency of coexistence of different gp195 alleles in a single isolate would be higher than that in the present study. The transmission of two genetically distinct organisms having different gp195 alleles into a mosquito vector via a bite, followed by zygote formation and meiosis wherein, should provide a good chance for the parasite to undergo intragenic recombination of gp 195 alleles. As a consequence, new alleles could appear. For example, intragenic recombination of alleles 1 and 2, the coexistence of which occurred in isolate 834 , at 
regions between blocks 2 and 4 and regions between blocks 4 and 6, creates alleles 3 and 4, and alleles 7 and 8, respectively. However, the frequency of intragenic recombination seems to be rare because neither allele 7 nor 8 has been found in the present study (see fig. 3).

The significance of this recombination remains unknown. Since sera from some individuals living in malaria endemic areas have antibodies which react with $\mathrm{gp} 195,{ }^{18}$ it may be that immunological, effector mechanisms invoked by the host select only some parasites possessing particular $\mathrm{gp} 195$ alleles in the circulation. It may be relevant to mention in this context that the recombination events are restricted to the 5 ' end of the gp195 gene. The N-terminus $80-83 \mathrm{kDa}$ fragment, which is one of the processed products of $\mathrm{gp} 195$, is shed from the merozoite surface upon entry into an erythrocyte and is therefore likely to be immunogenic. Thus, recombination at the 5 ' end could increase the chance of $P$. falciparum survival in the host circulation. Alternatively, it must also be considered that functional constraints of gp195 restrict only a few appropriate alleles. The function of $\mathrm{gp} 195$ is presently unknown. However, the protein or the processed products may play an important role in the erythrocyte invasion by the merozoite, because a monoclonal antibody against $\mathrm{gp} 195$ has been reported to block the invasion. ${ }^{\prime}$ It is intriguing to speculate that gp195 may serve as a ligand for the erythrocyte receptor of the merozoite. Evidence also indicates that there are possibly two kinds of erythrocyte receptors, glycophorin and an unidentified substance. ${ }^{19,20}$ Assuming that each of the dimorphic gp195 can interact only with one receptor, recombination at the 3' end between dimorphic gp195 alleles could code a protein that is incompatible with either of the receptors. Nevertheless, whatever the significance of the recombination is, common coexistence of different gp 195 alleles not only in a small malaria field but also in a single patient should be taken into account for future development of a malaria vaccine based on gp195. To date, no extensive search for $B$ cell and $T$ cell epitopes in gp195 has been conducted, though a few were found. ${ }^{21,} 22$ If immunodominant $B$ cell and/or $T$ cell epitopes were localized at variable peptide regions, a vaccine would have to incorporate the epitopes from the two dimorphic proteins which form the basis for the rest of the allelic variation.
Acknowledgments: We thank Tetsuo Yanagi and Michiko Matsuo for helping prepare the figures.

Financial support: Grant-in-Aid from the Ministry of Education, Science and Culture of Japan; Research grant from Osaka Institute of Technology; Hitachi Scholarship Foundation (Tokyo).

Authors' addresses: Somchai Jongwutiwes, Shusuke Nakazawa, Haruki Uemura, Hiroji Kanbara, Department of Protozoology, Institute of Tropical Medicine, Nagasaki University, Sakamoto-machi, Nagasaki 852, Japan. Kazuyuki Tanabe, Laboratory of Biology, Osaka Institute of Technology, Ohmiya, Asahi-ku, Osaka 535, Japan.

Reprint requests: Somchai Jongwutiwes, Department of Protozoology, Institute of Tropical Medicine, Nagasaki University, 12-4 Sakamoto-machi, Nagasaki 852, Japan.

\section{REFERENCES}

1. Holder AA, 1988. The precursor to major merozoite surface antigens: structure and role in immunity. Prog Allergy 41: 72-97.

2. Hall R, Hyde JE, Goman M, Simmons DL, Hope IA, Mackay M, Scaife J, Merkli B, Richle R, Stocker J, 1984. Major surface antigen gene of a human malaria parasite cloned and expressed in bacteria. Nature 311(5984): 379-382.

3. Cheung A, Leban J, Shaw AR, Merkli B, Stocker J, Chizzolini C, Sander C, Perrin LH, 1986. Immunization with synthetic peptides of a Plasmodium falciparum surface antigen induces antimerozoite antibodies. Proc Natl Acad Sci US A 83(21): 8328-8332.

4. Siddiqui WA, Tam LQ, Kramer KJ, Hui GS, Case SE, Yamaga KM, Chang SP, Chan EB, Kan SC, 1987. Merozoite surface coat precursor protein completely protects Aotus monkeys against Plasmodium falciparum malaria. Proc Natl Acad Sci U S A 84(9): 3014-3018.

5. Patarroyo ME, Romero $P$, Torres ML, Clavijo $P$, Moreno A, Mart:inez A, Rodr:iguez R, Guzman $F$, Cabezas E, 1987. Induction of protective immunity against experimental infection with malaria using synthetic peptides. Nature 328(6131): 629-632.

6. McBride JS, Newbold CI, Anand R, 1985. Polymorphism of a high molecular weight schizont antigen of the human malaria parasite Plasmodium falciparum. J Exp Med 161(1): 160180.

7. Tanabe K, Mackay M, Goman M, Scaife JG, 1987. Allelic dimorphism in a surface antigen gene of the malaria parasite Plasmodium falciparum. $J$ Mol Biol 195(2): 273-287.

8. Peterson MG, Coppel RL, McIntyre P, Lanford CJ, Woodrow G, Brown GV, Anders RF, Kemp DJ, 1988. Variation in the precursor to major merozoite surface antigens of Plasmodium falciparum. Mol Biochem Parasitol 27: 291-302.

9. Chang SP, Kramer KJ, Yamaga KM, Kato A, Case SE, Siddiqui WA, 1988. Plasmodium falcipa- 
rum: gene structure and hydropathy profile of the major merozoite surface antigen (gp195) of the Uganda-Palo Alto isolate. Exp Parasitol 67: $1-11$.

10. Certa U, Rotmann D, Matile H, Reber-Liske R, 1987. A naturally occurring gene encoding the major surface antigen precursor p190 of Plasmodium falciparum lacks tripeptide repeats. $E M B O$ J 6(13): 4137-4142.

11. Peterson MG, Coppel RL, Moloney MB, Kemp DJ, 1988. Third form of the precursor to the major merozoite surface antigens of Plasmodium falciparum. Mol Cell Biol 8: 2664-2667.

12. Meryman HT, Hornblower M, 1972. A method for freezing and washing red blood cells using a high glycerol concentration. Transfusion 12(3): 145-156.

13. Trager $W$, Jensen JB, 1976. Human malaria parasites in continuous culture. Science 193(4254): 673-675.

14. Tanabe K, Murakami K, Doi S, 1989. Plasmodium falciparum: dimorphism of the p190 alleles. Exp Parasitol 68(4): 470-473.

15. Sambrook J, Fritsch B, Maniatis T, 1989. Molecular Cloning, a Laboratory Manual. New York: Cold Spring Harbor Laboratory.

16. Creasy A, Fenton B, Walker A, Thaithong S, Oliveria S, Mutambu S, Walliker D, 1990. Genetic diversity of Plasmodium falciparum shows geographical variation. Am J Trop Med Hyg 42: 403-413.

17. Thaithong S, Beale GH, Fenton B, McBride J, Rosario V, Walker A, Walliker D, 1984. Clonal diversity in a single isolate of the malaria parasite Plasmodium falciparum. Trans $R$ Soc Trop Med Hyg 78(2): 242-245.

18. Muller HM, Fruh K, von Brunn A, Esposito F, Lombardi S, Crisanti A, Bujard H, 1989. Development of the human immune response against the major surface protein (Bp190) of Plasmodium falciparum. Infect Immun 57(12): 3765-3769.

19. Mitchell GH, Hadley TJ, McGinniss MH, Klotz FW, Miller LH, 1986. Invasion of erythrocytes by Plasmodium falciparum malaria parasites: evidence for receptor heterogeneity and two receptors. Blood 67(5): 1519-1521.

20. Hadley TJ, Klotz FW, Pasvol G, Haynes JD, McGinniss MH, Okubo Y, Miller LH, 1987. Falciparum malaria parasites invade erythrocytes that lack glycophorin A and B (MkMk). Strain differences indicate receptor heterogeneity and two pathways for invasion. JClin Invest 80(4): 1190-1193.

21. Sinigaglia F, Takacs B, Jacot $H$, Matile H, Pink JRL, Crisanti A, Bujard H, 1988. Nonpolymorphic regions of p190, a protein of the Plasmodium falicparum erythrocytic stage, contain both $T$ and B cell epitopes. J Immunol 140: 3568-3572.

22. Crisanti A, Muller HM, Hilbich C, Sinigaglia F, Matile H, McKay M, Scaife J, Beyreuther K, Bujard H, 1988. Epitopes recognized by human $T$ cells map within the conserved part of the gp190 of P. falciparum. Science 240: 13241326. 